\title{
The effects of vibration frequency variation on volleyball players' drop jump ability and postural control performance
}

\author{
Izzet KIRKAYA¹, Deniz SIMSEK², Hayri ERTAN² \\ ${ }^{1}$ Graduate School of Health Sciences Department of Physical Education and Sports, Anadolu University, Eskisehir, Turkey. \\ ${ }^{2}$ Facult of Sport Sciences, Anadolu University, Eskisehir, Turkey. \\ Address Correspondence to D. Simsek, deniz_yenigelen26@hotmail.com
}

\begin{abstract}
The aim of the performed study is to present the acute effect of the whole body vibration (WBV) stimuli on drop jump (DJ) and postural control performance. 13 athletes who play in Turkish Women Volleyball Second League voluntarily attended to the study $(22.3 \pm 2.6$ age; $60.72 \mathrm{~kg} \pm 1.74 \mathrm{~kg} ; 1.79 \mathrm{~cm} \pm 6.4 \mathrm{~cm}$ ). Athletes were randomly divided into three groups (group $1: \mathrm{n}=4: 30 \mathrm{~Hz}$; group 2: $n=5: 35$; group 3: $n=4: 40 \mathrm{~Hz}$ ). Before fatigue protocol, every participant's rest center of pressure- sway (CoP-sway) and DJ measurements on force plate were taken. Statistical whole body vibration measurements were practiced on Compex WINPLATE on different frequency $(30-35-40 \mathrm{~Hz})$, on high amplitude, in the manner that knee flexion angle is $120^{\circ}$ in 60 seconds. DJ breaking point which is determined for each athlete was applied as DJ jump height after vibration. Just after the fatigue protocol was completed, athletes' CoP- sway measurements after fatigue were practiced on force plate. Different frequency measurements taken to analyse the effects on DJ height of frequency at specific amplitudes were evaluated by ANOVA analysis. Based on variance homogeneity test, the Tukey or Tamhane methods were used for multiple comparisons. A statistical analysis with a paired Student's $t$ test was used to compare pre-vibration and post-vibration treatment values of jumping height at the DJ test. DJ performance is affected by WBV stimuli in different frequency, CoP- sway values and especially fatigue situation in AP direction ( $\mathrm{p}<0.05$ ). In conclusion, $30 \mathrm{~Hz}-35 \mathrm{~Hz}$ and $40 \mathrm{~Hz} \mathrm{WBV}$ stimuli has an acute effect on DJ performance beside that $35 \mathrm{~Hz}$ WBV definitely has the greatest effect.
\end{abstract}

Keywords: CoP sway, drop jump, postural control, fatigue.

\section{INTRODUCTION}

In order to evaluate the falling performance during training, different neuromuscular fatigue protocols are applied. Among these protocols, besides whole body and local vibration stimuli; fatigue measurements from getting just one muscle fiber tired to fatigue protocols to apply to even whole body are performed (5). With these measurements the situation that muscles cannot produce any force in wanted level is presented. In other words, fatigue concept is limited by a point called 'breaking point' and changed from person to person. When this point is come, fatigue abruptly occurs and the training cannot be continued. However, before this point is reached, many neurophysiologic mechanisms send many signals about arising fatigue in the body (4). Abrupt training ending is known as an adaption that neuromuscular fatigue lowers its own limits without any damage to the muscle itself and it is known as 'muscle wisdom'. Muscle wisdom limits membrane sensitiveness and correspondingly secretion of $\mathrm{Ca}^{2+}$ by limiting fatigue. This system occurs by central stimuli's sending 'decreasing production of force' feedback to active myofibrils in the direction of the information coming from peripheral afferents to the center (27). Developing fatigue basically depends on more than one reason. Though generally (i) decreasing rate of $\mathrm{Ca}^{2+}$ ion that secreted from sarcoplasmic reticulum (SR) have an important role in having fatigue situation. Because of the fact that decreasing synthesis pace of $\mathrm{Ca}^{2+}$ may affect the contractile mechanism, decreasing in produced force concordantly occurs. Short-term intense exercise weakens the contraction ability of muscle by making deterioration in the order of SR $\mathrm{Ca}^{2+}$ and causes deterioration in also routine of cross bridge by composing acidosis $(1,19)$. The other factor in arising fatigue is (ii) decreasing the participating rate of motor units that are supposed to contribute to the total committed work. Decreasing motor unit 
activities results in decreasing contraction race and failure in peripheral transfer. Decrease seen in the race of maximum voluntarily contraction will directly lower the force outcome (18).

Whole body vibration exercises that have gradually become popular get much interest especially among elite athletes to increase muscular performance and are densely used.7,8,9 In the performed studies, it is a known truth that whole body vibration (1) increases the muscle force $(3,6,8,11,16,17,22,23,24)$, (3) increases the jumping ability.

Because of the nature of volleyball sport branch, jumping is a typical example to see the effects of external forces on increasing the length of the muscle. The muscle, after the concentric contraction, contracts eccentrically. The combination of these eccentric and concentric movements composes the natural action of muscle called the stretch and shortening cycle (28). Exercises for the stretch and shortening cycle are used by athletes, trainers and conditioners to increase the performance (25). With jumping workout which takes place in these exercises, neuromuscular contraction and stretching workout have been done. Stretching and shortening cycle in lower extremity is a reflex movement in knee and ankle tendomuscular system as eccentric forward loading (contraction) in landing phase of jumping before concentric contraction in hanging phase. Stretch reflexion forms the most important mechanism underlying the stretch and shortening cycle (21). Due to the fact that frequency or/and amplitude place changes that are composed by WBV platform also cause some differences in muscle length and they are thought as an alternative method that is necessary to add to athletes' exercise programs. Based on these observations, the WBV exposure is supposed to enhance the height of maximal vertical jumping and postural control performance at a certain level. However, when the literature scanning was performed, any studies that the acute effect of whole body vibration on both volleyball players' jumping height and postural control was analyzed were not encountered. Therefore, it may be stated that little information about this subject is in scientific literature. The basic aim of this study is to analyze the acute effect of static WBV stimuli in elite female volleyball players to postural control and DJ performance. Concordantly, (1) more and more frequency of WBV stimuli, faster neuromuscular fatigue occurs and correspondingly the height of jumping decreases, (2) postural orientation during static WBV shows a direct relationship with the frequency of the given vibration stimuli so these two situations form the hypothesis of the performed study.

\section{MATERIAL \& METHOD}

\section{Participants}

13 athletes who play Turkish Women Volleyball 2nd League and do not have any contradictions (epilepsy, diabetes, galistone, renal calculus, acute inflammation, joint problems, cardiovascular diseases, joint implants, thrombosis, back problems like hernia and tumours) voluntarily participated to the study in the direction of producer's criteria related to WBV (22.3 \pm 2.6 age; $60.72 \mathrm{~kg} \pm 1.74 \mathrm{~kg} ; 1.79 \mathrm{~cm} \pm 6.4 \mathrm{~cm})$. The study was confirmed by Labor Local Ethics Committee. Consent form was signed by experimental before the participation.

\section{Study Design}

24 hours before the test procedure that will be applied, all participants were crisply kept away the activities that may spoil their daily biorhythm and are tiring. Participants applied the tests on the same day and at the same hour. In order to make each participant accord with the test procedure and equipment, DJ and CoP- sway measurements were checked by all of the participants one day before the real test as preliminary test and last test. To eliminate the effect of different shoes in vibration measurements, the measurements were practiced without shoes.

\section{The Drop Jump test}

Before the DJ test, the volunteer participants were wanted to warm up with jog and six repetitive with \% 60 density leg extension contractions and stretching with 3 sets. After the warm -up, the participants were wanted to go to the box that DJ would be practiced and put their hands to their hips (akimbo) and practiced the jumping when the 'jump' command was got. While the DJ was practiced, any commands about jumping technique were not given. The participants were merely wanted to practice the highest jumping as much as they can do. In order to not form the effect of order, DJ case heights were randomly determined. DJ case heights were checked maximum five times from 20 $\mathrm{cm}$ to $60 \mathrm{~cm}$ with $5 \mathrm{~cm}$ gaps and the place where the breaking height takes place was determined as DJ case height. DJ measurements were calculated by having been used $h=9,81 \times \mathrm{t}^{2} / 8$ and with the help of force platform (Type Kistler, 9281EA, Germany). 


\section{Vibration Data Acquisition}

Before the experimental protocol, in order to adapt to WBV feeling, a practicing session was applied to the athletes. The athletes were wanted to do static squat without any load with the position that knee flexion angle is $120^{\circ}$. By checking angle change in the knee joint angle with electronic goniometry, instant feedback was provided to the participants with computer monitor. Whole body vibration was practiced on Compex WINPLATE (Galileo 2000, Novotec Medical GmBH, Germany) in different frequency and in high amplitude $(4 \mathrm{~mm})$. All experiment conditions were 60sn length and the subjects started the exercise when they provided a comfortable squat movement on the platform. For each subject, signals appropriate to the foot span were placed on the vibration platform. During the session, the subject was only wanted to put their feet on the signals.

\section{CoPsway Acquisition}

The anteroposterior (AP), mediolateral (ML), components of the ground reaction forces of drop jump, rest and post CoP-sway values were measured at a sampling frequency of $2000 \mathrm{~Hz}$ using a Kistler force plate (Kistler, 9281EA, Germany) and normalized according to body weight. Data calculation formula is given in Table 1.

\section{Fatigue Protocol}

After resting CoP- sway was determined, the participants' DJ case height was found. The height of jumping that was practised from DJ case height was calculated using $\mathrm{h}=9,81 \times \mathrm{t} 2 / 8$ formula. As a fatigue protocol, 60 seconds vibration session was applied to the all groups in $4 \mathrm{~mm}$ amplitude in group $1=30 \mathrm{~Hz}$, group $2=35 \mathrm{~Hz}$ and group $3=40 \mathrm{~Hz}$ frequency. Just after the vibration session, athletes were wanted to practice the jumping from their own DJ case height. After having 60 seconds break, the other repetition was practiced. The DJ jumping height that had been reached before fatigue protocol was taken as breaking point and in the height that could not be reached the test was ended for the participants.

\section{Statistical Analysis}

Whole the statistical analysis was done via special statistics packet program (SPSS for Windows version 20; SPSS, Chicago, IL). Different frequency measurements taken to analyse the effects on Drop Jump height of frequency at specific amplitudes were evaluated by ANOVA analysis. Based on variance homogeneity test, the Tukey or Tamhane methods were used for multiple comparisons. A statistical analysis with a paired Student's t test was used to compare pre-vibration and post-vibration treatment values of jumping height at the DJ test. The variability of the CoP-sway in the A/P and M/L directions was calculated from the force plate data. Data are presented as means \pm SD. Statistical analysis was done using t-test. The statistical significance level was accepted as $\mathrm{P}<0.05$.

Table 1. Force plate calculation formulas.

\begin{tabular}{|c|c|c|}
\hline & & $\mathrm{P}^{\mathrm{X}} \overbrace{\mathrm{Z}}^{-\mathbf{Y}}$ \\
\hline Output signal & Channel & Description \\
\hline $\mathrm{f} \times 12$ & 1 & Force in X-direction measured by sensor $1+$ sensor 2 \\
\hline $\mathrm{f} \times 34$ & 2 & Force in X-direction measured by sensor $3+$ sensor 4 \\
\hline fy14 & 3 & Force in Y-direction measured by sensor $1+$ sensor 4 \\
\hline fy 23 & 4 & Force in Y-direction measured by sensor $2+$ sensor 3 \\
\hline $\mathrm{fz} 1 \ldots \mathrm{fz} 4$ & $5 \ldots 8$ & Force in $\mathrm{Z}$ direction measured by sensor $1 \ldots 4$ \\
\hline Parameter & Calculation & Description \\
\hline Fx & $=\mathrm{f} \times 12+\mathrm{f} \times 34$ & Medio-lateral force \\
\hline Fy & $=$ fy $14+$ fy 23 & Anterior-posterior force \\
\hline $\mathrm{Fz}$ & $=\mathrm{fz} 1+\mathrm{fz} 2+\mathrm{fz} 3+\mathrm{fz} 4$ & Vertical force \\
\hline
\end{tabular}




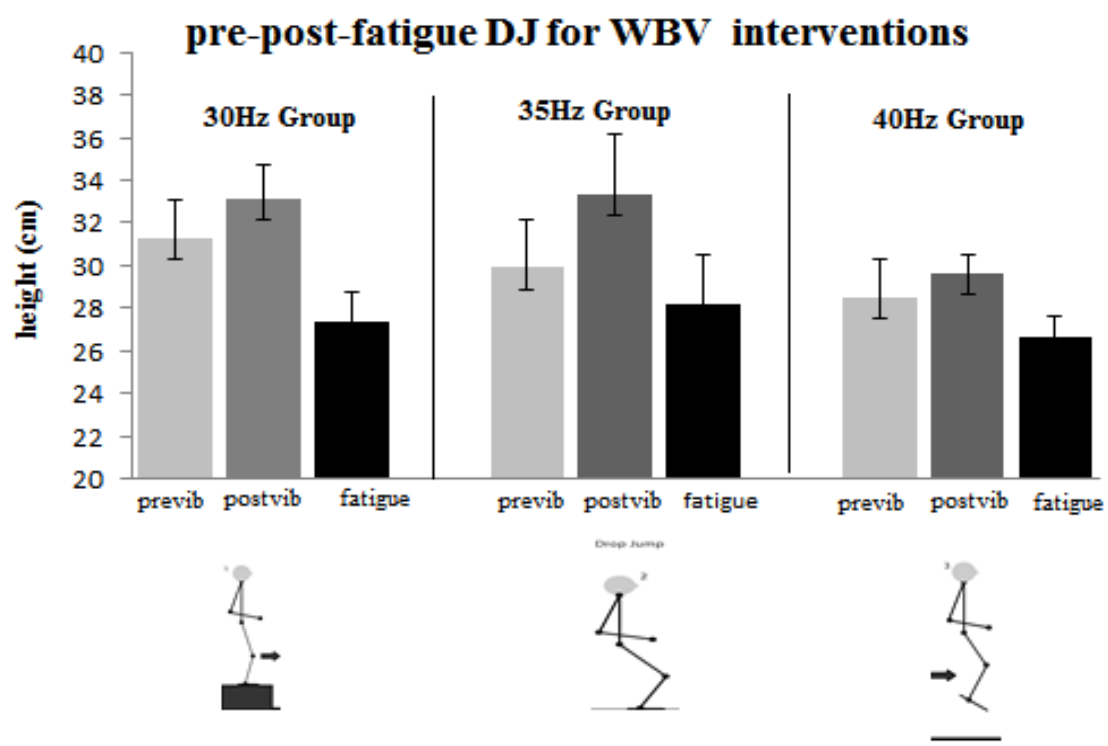

DJ: Drop Jump, WBV: Whole Body Vibration

Note: pre vib-30Hz-35Hz: ${ }^{\circledR}$, pre vib-35Hz-40Hz:¥, pre vib-30Hz40Hz: $\infty$;post vib-30Hz-35Hz: €, Post vib-30Hz-40Hz: ỏ, Post vib-35Hz-40Hz:£ ; fatigue- $30 \mathrm{~Hz}-35 \mathrm{~Hz}: \mu$, fatigue- $30 \mathrm{~Hz}-40 \mathrm{~Hz}: \beta$, fatigue- $35 \mathrm{~Hz}-40 \mathrm{~Hz}$ : $\phi(\mathrm{p}<0.05)$.

Figure 1. Pre- post fatigue drop jump height for whole body interventions.

Table 2. The Jumping heights of $30 \mathrm{~Hz}-35 \mathrm{~Hz}-40 \mathrm{~Hz}$ groups in the condition of fatigue and peak, pre vibration and the repetition table that these heights were reached.

\begin{tabular}{lccc}
\hline Group & $30 \mathrm{~Hz}$ & $35 \mathrm{~Hz}$ & $40 \mathrm{~Hz}$ \\
\hline & & & \\
Pre Vib DJ Jump Height (cm) & 31.25 & 31.10 & 28.50 \\
Peak DJ Jump Height (cm) & 33.11 & 32.52 & 29.62 \\
Repetition Number of Peak Jump Height & 3 & 7 & 4 \\
Jump Height Condition of Fatigue (cm) & 27.32 & 28.19 & 26.69 \\
Repetition Number When Fatigue Occured & 8 & 11 & 7 \\
\end{tabular}

\section{RESULTS}

In the study that the acute effect of static WBV stimuli to postural control and DJ performance in elite women volleyball players was analyzed, (1) pre -static WBV stimuli and post - static WBV stimuli and drop jump heights that were reached in fatigue condition (mean values $\pm \mathrm{SD}$ ) and (2) information that belongs to $\mathrm{CoP}$ - sway measurements pre -static WBV stimuli and post - static WBV stimuli (mean values \pm SD) are seen in Figure 1 and Table 2

When the Figure 1 and Table 2 are analyzed, DJ jump height of $30 \mathrm{~Hz}$ group before WBV is $31.25 \pm 1.87 \mathrm{~cm}$. The jumping height that emerged from the acute effect of WBV is $33.11 \pm 1.63 \mathrm{~cm}$ and this height was gained in the $3^{\text {rd }}$ repetition. Fatigue was reached in the $8^{\text {th }}$ repetition and jumping height that arose in fatigue condition is $27.32 \pm 1.39 \mathrm{~cm}$. The application of acute $30 \mathrm{~Hz}$ WBV caused $1.86 \mathrm{~cm}$ rise in DJ jumping height. The analysis with a paired $t$ test showed a significant difference between the pre and post vibration treatment measures $(\mathrm{p}<0.05)$.

Pre WBV DJ jumping height of $35 \mathrm{~Hz}$ group is $29.90 \pm 2.23 \mathrm{~cm}$. The jumping height that rose from acute effect of WBV is $33.32 \pm 2.86 \mathrm{~cm}$ and this height was obtained in the $7^{\text {th }}$ repetition. Fatigue was reached in the $11^{\text {th }}$ repetition and the jumping height that occurred in this repetition is $28.19 \pm 2.27 \mathrm{~cm}$. Acute $35 \mathrm{~Hz}$ WBV application caused $3.42 \mathrm{~cm}$ rise in DJ jumping height. The analysis with a paired $t$ test showed a significant difference between the pre and post vibration treatment measures $(p<0.05)$. 
pre and post COP sway for static WBV interventions

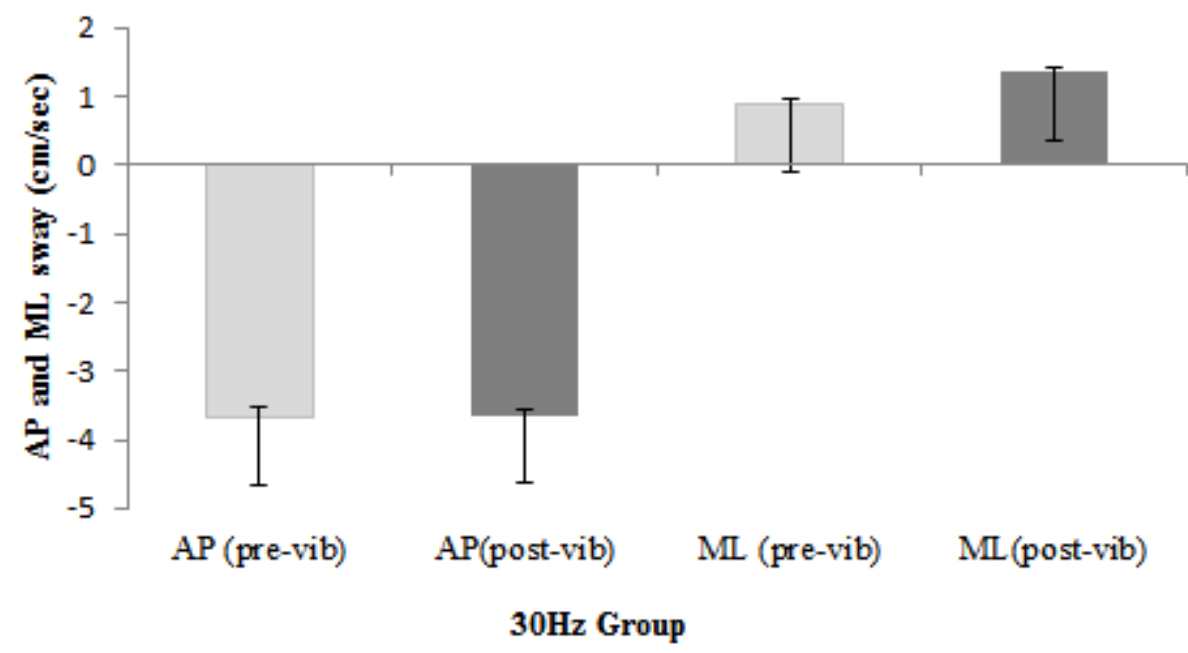

Significant differences from pre-vib are identified by ${ }^{*}(\mathrm{p}<0.05)$. Error bars are SD. AP: anterior-posterior; ML: medio-lateral

Figure 2. Pre - post CoPsway for static whole body interventions for $30 \mathrm{~Hz}$ group.

Pre vibration DJ jumping height of $40 \mathrm{~Hz}$ group is $28.50 \pm 1.79 \mathrm{~cm}$. The jumping height that emerged from the acute effect of WBV is $29.62 \pm 0.93 \mathrm{~cm}$ and this height was acquired in the $4^{\text {th }}$ repetition. Fatigue was reached in the $7^{\text {th }}$ repetition and the jumping height that occurred in fatigue condition is $26.69 \pm 0.90 \mathrm{~cm}$. The findings of the study provide that acute $40 \mathrm{~Hz}$ WBV application caused $1.32 \mathrm{~cm}$ rise in DJ jumping height. However, this rise was not found important in terms of statistical $(p>0.05)$.

Comparison tests show there are not any statistically significant differences between pre-post and fatigue DJ jumping height of frequency groups $(\mathrm{p}>0.05)$.

In the Figure 2- while the athletes' resting and fatigue CoP-sway measurements on the force plate were determined, AP axis presents (-) forward (+) back swinging; ML axis presents (-) left (+) right swinging.

On the AP axis Pre CoP-sway value of $30 \mathrm{~Hz}$ group shows $3.6 \pm 0.14 \mathrm{~cm} / \mathrm{s}$ shows forward swinging; Post CoP- sway value shows $3.5 \pm 0.07 \mathrm{~cm} / \mathrm{s}$ forward swinging. Pre CoP- sway value on the ML axis shows $1 \pm 0.09 \mathrm{~cm} / \mathrm{s}$ right swinging, Post CoP- sway value shows $1.5 \pm 0.07 \mathrm{~cm} / \mathrm{s}$ left swinging.

$35 \mathrm{~Hz}$ group's Pre CoP- sway value on AP axis shows $2.27 \pm 0.16 \mathrm{~cm} / \mathrm{s}$ forward; Post CoP- sway value shows $2.46 \pm 0.14 \mathrm{~cm} / \mathrm{s}$ forward swinging. Pre CoP sway value on $\mathrm{ML}$ axis shows $0.17 \pm 0.11 \mathrm{~cm} / \mathrm{s}$ right swinging, Post CoP- sway value shows $-0.5 \pm 0.63$ $\mathrm{cm} / \mathrm{s}$ left swinging.

While $40 \mathrm{~Hz}$ group's Pre CoP-sway value on the AP axis is close to zero and Post CoP -sway value shows $3.06 \pm 0.13 \mathrm{~cm} / \mathrm{s}$ forward swinging, Pre CoP - sway value on ML axis shows $0.15 \pm 0.08 \mathrm{~cm} / \mathrm{s}$ left swinging, Post CoPsway value shows right swinging.

\section{DISCUSSION}

In this study that is trying to explain how whole body vibration needs to be used in DJ movement as fatigue protocol and what sort of effects this presents in $\mathrm{Hz}$ groups, pre and post CoPsway and DJ jumping height were used as a fatigue marker with the aim of showing the emergence of the effect of fatigue.

Cairns et al. (5) have said in their study that the applied fatigue protocol is supposed to gradually show the decrease in the total work that arises in the number, duration, speed of contraction and conclusion by making the load that is applied in voluntary contraction stable. In the same study they have emphasized that fatigue symptoms are managed to be defined with EMG signals or the increasing pressure in muscle or whatever the work is done decline in the observed total work. In our study by protecting Cairns et al's $\mathrm{s}^{1}$ study, we limited emerging fatigue condition as jumping heights that is below the post $\mathrm{DJ}$ jumping heights. 


\section{pre and post COP sway for static WBV interventions}

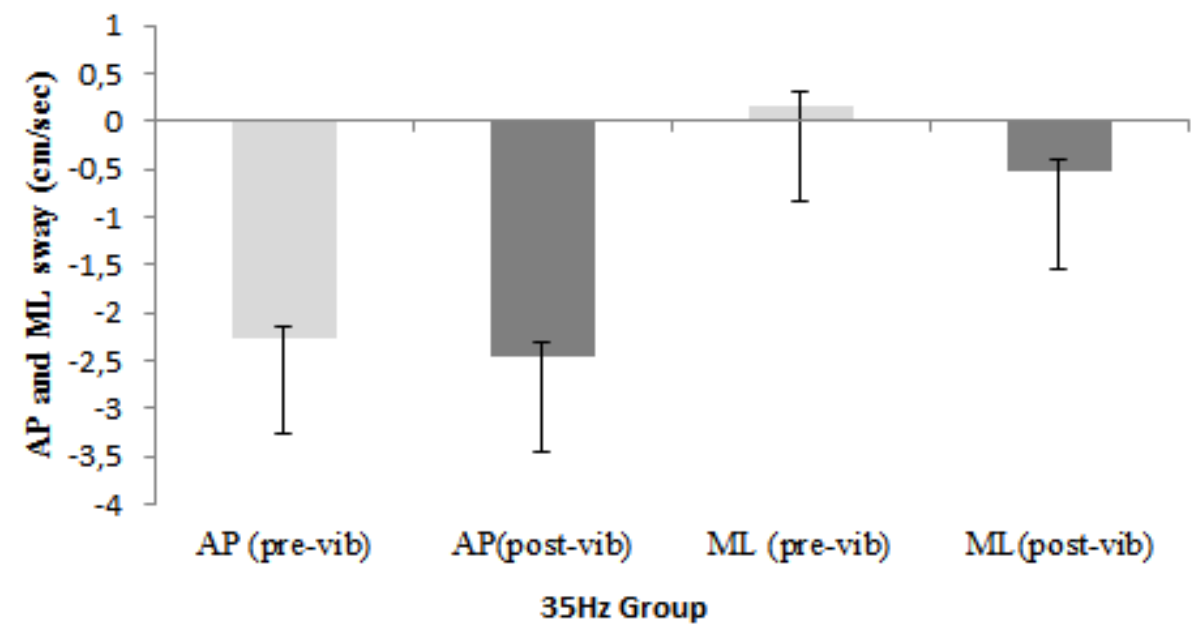

Significant differences from pre-vib are identified by * $(\mathrm{p}<0.05)$. Error bars are SD. AP: anterior-posterior; ML: medio-lateral Figure 3. Pre - post CoPsway for static whole body interventions for $35 \mathrm{~Hz}$ group.

pre and post COP sway for static WBV interventions

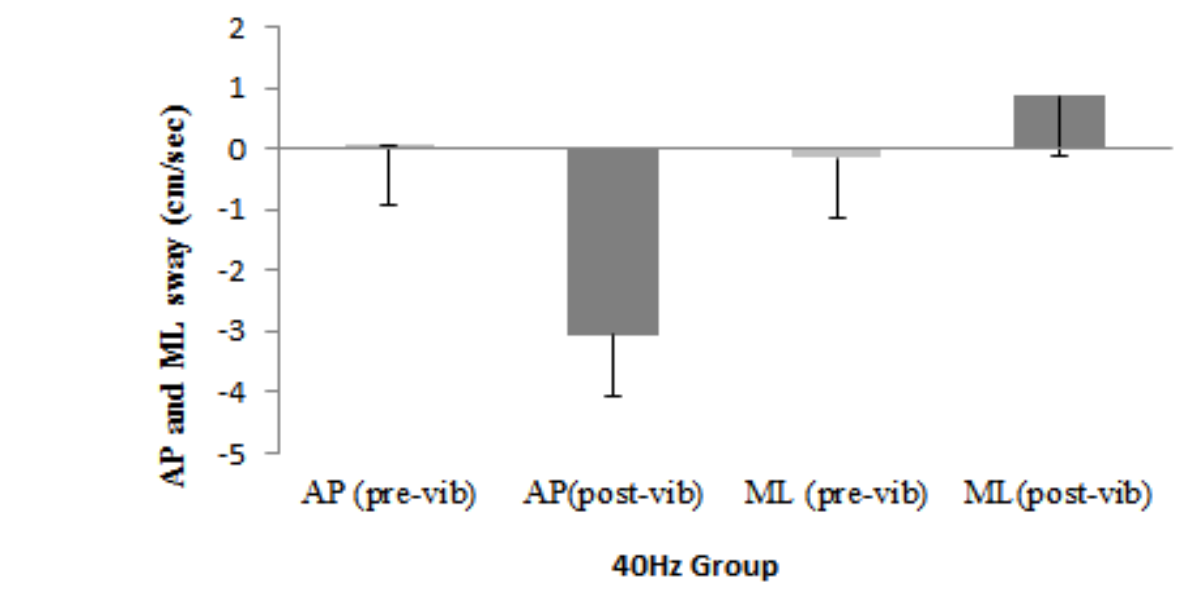

Significant differences from pre-vib are identified by * $(\mathrm{p}<0.05)$. Error bars are SD. AP: anterior-posterior; ML: medio-lateral

Figure 4. Pre - post copsway for static whole body interventions for $40 \mathrm{~Hz}$ group.

Gimmon et al. (14) applied fatigue protocol to plantar flexor and the muscles in the upper extremity. In the CoP- sway measurements that they practiced after fatigue protocol which they especially applied to plantar flexor muscles, statistically significant substitution on anteroposterior (AP) and mediolateral (ML) axis. Gribble \& Hertel (15) stated that during fatigue, neuromuscular system provides necessary muscular tensions, when fatigue increases, these tensions run short and correspondingly irretrievable substitutions in $\mathrm{CoP}$ are observed. Moreover, during fatigue, decreasing the speed of transferring afferent signals causes delay in efferent signals. This limits that muscle regression resists to $\mathrm{CoP}$ substitution.
After WBV stimuli that we applied, especially in CoPsway measurement of $40 \mathrm{~Hz}$ group, approximately $3 \mathrm{~cm}$ substitution was observed on $\mathrm{AP}$ axis and on ML axis approximately $0,5 \mathrm{~cm}$ substitution was. As a cause of this, it can be showed that fatigue protocol ruins kinetic chain movement that is from proximal to distal and proprioceptive stimuli causes to affect balance on postural muscles of continuous activity. Especially in the condition of fatigue, it can be said that having regression on $\mathrm{AP}$ axis is because of delay on neurologic transfer of ankle joint. Briefly, decreased motor outputs affect the sensibility of proprioceptors negatively (26). Post fatigue protocol in the CoPsway measurements that they applied in unipedal posture; they observed the 
greatest differences on AP axis. Even if CoPsway that we applied in the study is bipedal, the observed greatest substitution occurred on $\mathrm{AP}$ axis. This supports that on postural control, procsimal muscles have more effects than distal muscles. This condition was explained in the literature with that swinging on AP axis is much more than on ML axis (15).

Chen et al. (7) stated that in drop jump that they applied to $13 \mathrm{~Hz}$ and $50 \mathrm{~Hz}$ groups after whole body static squat that was in $3 \mathrm{~mm}$ amplitude and the knees' angle was $150^{\circ}$, both groups reached to peak jump height in $4^{\text {th }}$ jump. After whole body vibration that we applied, the one that is the closest to peak jump values which we gained in DJ movement is seen as $40 \mathrm{~Hz}$ group.

Chuang \& Chen (9) analyzed the differences in drop jump movement after $60 \mathrm{sec}$. whole body vibration that they applied to $40 \mathrm{~Hz}$ group in $2 \mathrm{~mm}$ amplitude with 3 and 7 sets. While in the $3^{\text {rd }}$ set a $1.63 \mathrm{~cm}$ rise was seen, after $7^{\text {th }}$ set a $2.41 \mathrm{~cm}$ rise was seen. Even though the protocol that they practiced is not fatigue protocol, it has some same sides with the protocol of our study. $30 \mathrm{~Hz}$ group reached to peak DJ height after 3 sets and the rise is $1.63 \mathrm{~cm}(\mathrm{p}<0.043)$. In $35 \mathrm{~Hz}$ group, peak DJ heights were appeared after $7^{\text {th }}$ repetition and the rise is $2.86 \mathrm{~cm}(\mathrm{p}<0.043) .40 \mathrm{~Hz}$ group entered to fatigue in the $7^{\text {th }}$ repetition and their DJ heights rose $1.32 \mathrm{~cm}(\mathrm{p}>0.150)$.

Cnieairns et al. (2013) stated that in DJ tests that they applied whole body vibration in $45 \mathrm{~Hz} 5 \mathrm{~mm}$ and $30 \mathrm{~Hz} 5 \mathrm{~mm}$, high vibration frequencies form more contact time with the ground and DJ jump height rise more. However, in our study it is seen that the high frequency group $(40 \mathrm{~Hz})$ had the lowest jump height rise $(1.32 \mathrm{~cm})$ that forms from acute effect and entered to fatigue the most quickly (after $7^{\text {th }}$ repetition).

In the literature, there are a number of methods as fatigue protocol essays and markers. Nevertheless these methods could not gain a certain provision in determining fatigue protocol. (1) 'In the condition of fatigue, postural swinging rises.' thesis which is our one number hypothesis was accepted by observing rises in CoP sway values of $\mathrm{A} / \mathrm{P}$ and $\mathrm{M} / \mathrm{L}$ axis. (2) When WBV stimuli frequency rises, faster neuromuscular fatigue occurs and correspondingly the height of the jump decreases. Apart from the peak DJ jump heights that form from acute effect of vibration after fatigue protocol, we can indicate that in different frequency $35 \mathrm{~Hz}$ and $4 \mathrm{~mm}$ amplitude values provide much more benefits. In DJ studies that were done with different case heights, Peng ${ }^{27}$ have said that practicing DJ movement from high case is not different from jumping from a low case in terms of biceps femoris and rectus femoris. Because of the fact that we decided case heights according to the participants' DJ breaking heights in our study, this topic was not mentioned. In the next studies the jumps that are done from different case heights can be done according to fatigue protocol that we applied and muscular activations can be observed with the help of electromyography. After acute vibration, the rise that is observed in DJ jump height has more than one cause. Some of these are commented as frequency and amplitude of vibration, tissue hardness or bloodstream of metabolism changes hydrodynamics positively. With the aim of minimizing these affects, it is suggested to use stable frequency, stable vibration amplitude and control group. Isometric contractions in isokinetic dynamometer or concentric-concentric contractions with the help of isokinetic dynamometer are suggested in order to evaluate CoPsway substitutions and specifically getting tired of muscles even if being the most substitutions on $\mathrm{AP}$ axis in fatigue protocol that occurred by being used WBV shows that postural muscles get tired much more. Therefore it can be observed how activation the joints follow under fatigue conditions by minimizing the variables.

\section{REFERENCES}

1. John Booth, Michael J. McKenna, Patricia A. Ruell, Tom H. Gwinn, Glen M. Davis, Martin W. Thompson, Alison R. Harmer, Sandra K. Hunter, John R. Sutton. Impaired calcium pump function does not slow relaxation in human skeletal muscle after prolonged exercise. The American Physiological Society, 1997; 0161-7567.

2. Bosco C, Cardinale M, Tsarpela O. Influence of vibration on mechanical power and electromyogram activity in human arm flexor muscles. European Journal of Applied Physiology, 1999; 79: 306-311.

3. Bosco C, Iacovelli M, Tsarpela O, Cardinale M, Bonifazi M, Tihanyi J, Viru M, De Lorenzo A, Viru A. Hormonal responses to whole-body vibration in men. European Journal of Applied Physiology, 2000- 81, 449-454

4. Boyas S, Guevel A. Neuromuscular fatigue in healthy muscle: underlying factors and adaptation mechanisms. Ann Phys Rehabil Med, 2011; 54(2): 88-108.

5. Cairns SP, Knicker AJ, Thompson MW, Sjøgaard G. Evaluation of models used to study neuromuscular fatigue. Exerc Sport Sci Rev, 2005; 33: 9-16.

6. Cardinale $\mathrm{M}$, Wakeling J. Whole body vibration exercise: Are vibrations good for you? British Journal of Sports Medicine, 2005; 39: 585-589.

7. Chen WC, Huang MY, Shiang TY. Short-term effects of different whole body vibration frequencies on the shallow drop jump in sprinter. Journal of Biomechanics, 2006; 39: 565. 
8. Cheung WH, Mok HW, Qin L, Sze PC, Lee KM, Leung KS. High frequency whole-body vibration improves balancing ability in elderly women. Archives of Physical Medicine and Rehabilitation, 2007; 88: 852-857.

9. Chuang TY, Chen WC. The effects of drop jump performance of different test timing after varied vibration stimulus. Journal of Biomechanics, 2007; 40 DOI: 10.1016/S0021-9290(07)70620-6.

10. Cochrane DJ, Stannard SR. Acute whole body vibration training increases vertical jump and flexibility performance in elite female field hockey players. British Journal of Sports Medicine, 2005; 39: 860-865.

11. Delecluse C, Roelants M, Verschueren S. Strength increase after whole-body vibration compared with resistance training. Medicine and Science in Sports and Exercise, 2003; 35: 1033-1041.

12. Fernandes IA, Kawchuk G, Bhambhani Y, Gomes PSC. Does whole-body vibration acutely improve power performance via increased short latency stretch reflex response? Journal of Science and Medicine in Sport / Sports Medicine Australia, 2013; 16(4): 360-4. doi:10.1016/j.jsams.2012.08.010

13. Fuglevend AJ, Keen DA. Re-evaluation of muscle wisdom in the human adductor pollicis using physiological rates of stimulation. J Physiol 2003; 549(3): 865-75.

14. Gimmon Y, Rimer R, Oddson L, Melzer I. The effect of plantar flexor muscle fatigue on postural control. J Electromyogr Kinesiol, 2011. 21(6):922-928.

15. Gribble PA, Hertel J. Effect of hip and ankle muscle fatigue on unipedal postural control. Journal of electromyography and kinesiology : official journal of the International Society of Electrophysiological Kinesiology, 2004; 14(6): 641-6.)

16. Luo J, McNamara B, Moran K. The use of vibration training to enhance muscle strength and power. Sports Medicine, 2005; 35: 23-41.

17. Mester J, Spitzenfeil P, Schwarzer J, Seifriz F. Biological reaction to vibration-implications for sport. Journal of Sports Science and Medicine, 1999; 2: 211-226.

18. Nielsen OB, Ortenblad N, Lamb GD, Stephonson DG. Excitability of the T-tubular system in rat skeletal muscle:
Kirkaya et al. 2015

roles of $\mathrm{K}^{+}$and $\mathrm{Na}^{+}$gradients and $\mathrm{Na}^{+}-\mathrm{K}^{+}$pump activity. $\mathrm{J}$ Physiol, 2004; 557(1): 133-146.

19. Pedersen TH, Nielsen OB, Lamb GD, Stephenson DG. Intracellular acidosis enhances the excitability of working muscle. Science, 2004; 305(5687): 1144-1147.

20. Peng HT. Changes in biomechanical properties during drop jumps of incremental height. Journal of Strength and Conditioning Research, 2011; 25(9): 2510-2518.

21. Rasorch R. The Different Muscle-Energetics during Shortening and Stretch. Int J Mol Sci, 2011; 12: 2891-2900.

22. Roelants M, Delecluse C, Verschueren SM. Whole-bodyvibration training increases knee-extension strength and speed of movement in older women. Journal of the American Geriatrics Society, 2004; 52: 901-908.

23. Ruiter CJ, Van Raak SM, Schilperoort JV, Hollander AP, de Haan A. The effects of 11 weeks whole body vibration training on jump height, contractile properties and activation of human knee extensors. European Journal of Applied Physiology, 2003; 90: 595-600

24. Torvinen $\mathrm{S}$, Kannus $\mathrm{P}$, Sievanen $\mathrm{H}$, Jarvinen TAH, Pasanen M, Kontulainen S, Jarvinen TLN, Jarvinen M, Oja P, Vuori I. Effect of a vibration exposure on muscular performance and body balance. Randomized cross-over study. Clinical Physiology and Functional Imaging, 2002; 22: 145-152.

25. Toumi H, Poumarat G, Best TM, Martin A, Fairclough J, Benjamin M. Fatigue and muscle-tendon stiffness after stretch-shortening cycle and isometric exercise. 2006 Applied Physiology, Nutrition, and Metabolism, 2006; 31(5): 565-572

26. Vuillerme N, Forestier N, Nougier V. Attentional demands and postural sway: the effect of the calf muscles fatigue. Medicine and Science in Sports and Exercise, 2002; 34(12): 1907-1912.

27. Windhorst U. Muscle proprioceptive feedback and spinal networks. Brain Res Bull, 2007; 73(4-6): 155-202.

28. Zatsiorsky V. Biomechanics of strength and strength training. In: Strength and power in sport. Ed: Komi P.V., editor. Oxford: Blackwell Scientific Publications; 2005: 439487. 